\title{
Depressive symptoms and pragmatic rehabilitation for chronic fatigue syndrome
}

\author{
A. J. Wearden, G. Dunn, C. Dowrick and R. K. Morriss
}

\section{Background}

Previous research has suggested that depressed mood may predict outcome and moderate response to treatment in chronic fatigue syndrome, although findings have differed between studies.

\section{Aims \\ To examine potential moderators of response to pragmatic rehabilitation $v$. general practitioner treatment as usual in a recent randomised trial for patients with chronic fatigue syndrome in primary care (IRCTN74156610).}

\section{Method}

Simple regressions, with weighting adjustments to allow for missing data, were calculated. Demographic, medical and psychological variables, and treatment arm, were entered separately and as an interaction term. The outcome variable in each case was change in Chalder Fatigue Scale scores, from baseline to 1-year follow-up, our primary outcome point.

\section{Results}

Longer illness durations predicted poorer outcome across the two treatment arms. For patients allocated to pragmatic rehabilitation compared with those allocated to treatment as usual, higher levels of depressive symptoms at baseline were associated with smaller improvements in fatigue $(P=0.022)$.

\section{Conclusions}

For patients in primary care with higher levels of depressive symptoms, either more intensive or longer pragmatic rehabilitation, or cognitive-behavioural therapy, may be required in order to show a significant improvement in fatigue.

\section{Declaration of interest}

None.
People with chronic fatigue syndrome (also known as myalgic encephalomyelitis; CFS/ME) experience severe, disabling fatigue plus other symptoms that cannot be accounted for by alternative medical diagnoses. ${ }^{1,2}$ Several systematic reviews ${ }^{3-7}$ and a recent large-scale treatment trial ${ }^{8}$ have shown that cognitive-behavioural therapy (CBT) and graded exercise therapy (GET), both of which encourage carefully graded increases in activity, are effective in reducing fatigue and improving functioning. The majority of trials for CFS have taken place in specialist or secondary care settings; in the UK, however, there have been calls for the management of patients with CFS/ME in primary care, with referral to specialist care only when needed. ${ }^{9,10}$ The recommendation for management in primary care reflects the scarcity of accessible secondary care treatment resources rather than a firm evidence base for primary care management of CFS/ME. ${ }^{11,12}$ We recently carried out a randomised controlled trial (RCT) of a treatment that we called pragmatic rehabilitation for patients in primary care with CFS/ME - the FINE trial (IRCTN74156610). ${ }^{13}$ Pragmatic rehabilitation was originally developed and tested in a hospital setting, ${ }^{14}$ but in the FINE trial it was delivered in patients' homes over an 18-week period by three specially trained general nurses. The treatment has elements in common with both CBT and GET but differs from them in that it starts with the explicit delivery of an explanatory model for patients' symptoms. The model focuses on factors that may be maintaining fatigue and activity limitations, explaining the roles of cardiovascular and muscular deconditioning, disturbed sleep-wake cycles, hypothalamic-pituitary-adrenal axis dysregulation, and the somatic manifestations of arousal. Patient and therapist then collaborate to design a rehabilitation programme based on addressing these factors.

In the FINE trial, pragmatic rehabilitation was compared with a non-directive counselling treatment, called supportive listening, and with treatment as usual by the general practitioner (GP). Although supportive listening was not effective in improving our primary outcomes (fatigue and physical functioning) or any of our secondary outcomes (depression, anxiety and sleep problems), patients allocated to pragmatic rehabilitation showed significant improvements in fatigue, depression and sleep problems at the end of treatment when compared with patients allocated to GP treatment as usual. ${ }^{13}$ These effects of pragmatic rehabilitation were modest in size and attenuated over the 1-year follow-up period, although only marginally in the case of fatigue. ${ }^{13,15}$ None of our treatments produced significant improvements in physical functioning. For a fuller report of rationale, design and findings of the FINE trial, see Wearden et al. ${ }^{16}$

Pragmatic rehabilitation was less effective in the primary care FINE trial than it had been in the previous secondary care trial, ${ }^{17}$ with smaller effects on fatigue scale scores and fewer patients recovering. In our original report, we proposed a number of possible reasons for this difference, among them the inclusion of severely affected non-ambulatory patients with high levels of functional limitations who would have been excluded from a secondary care trial, the inclusion of patients with comorbidities that may have complicated treatment and the inclusion of patients with long illness durations. ${ }^{13}$ When considering the findings from treatment trials, one may be interested in factors which are associated with better outcome in the sample as a whole (usually termed predictors of outcome). More often, it is important to know what factors may modify the efficacy of each treatment (usually termed moderators). Finally, it is important to determine how, or through what mechanisms, the treatment has its effects (usually termed mediators). ${ }^{18}$ The purpose of the present study was to examine factors measured at baseline that may have moderated response to pragmatic rehabilitation treatment in the FINE trial. We focused on the primary outcome for which treatment was effective, namely fatigue, and on our primary outcome point, 70 weeks. Mediators of treatment outcome will be reported in another paper. 
In addition to the potential moderators of response to pragmatic rehabilitation outlined earlier (illness severity and disability, comorbidities and illness duration), here we considered factors that had been shown to moderate response to treatment in the previous secondary care study - that is, level of symptoms of depression and anxiety, being in receipt of benefits and membership of a local self-help group. ${ }^{17}$ We also examined the effect of disturbed sleep at the start of the trial, reasoning that sleepiness may have affected the patients' ability to undertake graded activity and that spending therapy time regularising sleep patterns could have delayed the effect of other elements of pragmatic rehabilitation treatment. Finally, we examined the potential effects of age and gender. In our original report ${ }^{13}$ we noted that nurses encountered social barriers to treatment when delivering therapy in patients' homes. Although we did not have a measure of social problems, in accordance with our protocol, ${ }^{16}$ we examined whether the level of social support available to patients moderated their response to treatment. The FINE study inclusion criteria required that patients fulfilled the Oxford research diagnostic criteria. ${ }^{1}$ In order to assess the possibility that different diagnostic criteria define different groups who may respond differently to pragmatic rehabilitation treatment, we also examined whether fulfilment of the Fukuda ${ }^{2}$ or London $\mathrm{ME}^{19}$ criteria moderated response to treatment. The choice of potential moderator variables for this study was guided by the literature and, with the exception of sleep scale scores, specified in our protocol and analysis plan. ${ }^{19}$

\section{Method}

\section{Participants and trial design}

Participants were referred to the trial by their GPs. In total, 296 patients aged 18 and over who fulfilled the Oxford criteria for chronic fatigue syndrome ${ }^{1}$ and other study criteria $^{16}$ were randomly allocated to one of three treatment arms. Patients were randomised individually, after stratification on two factors whether the patient was ambulatory or not, and whether the patient fulfilled London ME criteria or not. The three treatment arms were pragmatic rehabilitation or supportive listening, both delivered in patients' homes by one of three specially trained general nurses, or treatment as usual from the patient's GP. Treatment lasted for 18 weeks.

\section{Assessments}

Patients were assessed prior to entry into the trial (baseline, week 0 ) within 2 weeks of the end of treatment (post-treatment, week 20) and at 1-year follow-up (week 70). All assessments were administered in the patients' homes by research assistants who were masked to the patient's treatment allocation. At each assessment, patients were interviewed and completed a set of self-report questionnaire measures. They also undertook a timed step test. ${ }^{20}$ Full details of all trial assessments are given elsewhere ${ }^{13,16}$ and only those measures used in the current study are detailed here.

\section{Outcomes}

The primary outcome point for the FINE trial was 70 weeks, and the present study focuses on this outcome point. The outcome measure was change in fatigue from baseline to 1-year follow-up. Fatigue was measured using the 11-item Chalder Fatigue Scale. ${ }^{21}$ For the present set of analyses, each item was scored $0,1,2$ or 3 , and the 11 items summed to produce a total scale score varying from 0 to 33 . Change scores were calculated by subtracting 70-week scores from baseline scores.

\section{Potential moderators}

All potential moderator variables were measures taken at the baseline assessment. Measures of illness severity and disability were: patient-reported ambulatory status, defined as use of a mobility aid on most days (yes/no); scores on questions relating to mobility, self-care and usual activities (each scored 0 - no problems, 1 - some problems and 2 - severe problems, and treated as a categorical variable) from the EQ-5D, ${ }^{22}$ and scores on the Short-Form 36-item questionnaire (SF-36) physical functioning scale, ${ }^{23}$ calculated as a percentage. Sleep problems were measured using the four items of the Jenkins Sleep Scale. ${ }^{24}$ At baseline assessment, the number of medical comorbidities reported by patients was recorded. Illness duration in months at baseline was taken from patient report.

Patients were asked whether they were a member of a local CFS/ME support group (yes/no). Levels of anxiety and depressive symptoms were measured using the summed scores on the seven items of each of the two Hospital Anxiety and Depression Scale (HADS) $;{ }^{25}$ in addition, the two scales were summed to produce a HADS total score. Fulfilment of diagnostic criteria for any depressive disorder was obtained from the Structured Clinical Interview for DSM-IV-TR Axis I Disorders (SCID-I). ${ }^{26}$ Social support was measured using the three-item Oslo Social Support Scale. ${ }^{27}$ The first question asks 'How many people are so close that you can count on them if you have serious personal problems' (none, 1 or 2, 3-5, more than 5 - treated as an ordinal scale), followed by two questions answered on five-point Likert-type scales, with higher scores indicating less support: 'How much concern do people show in what you are doing?', and 'How easy is it to get practical help from neighbours if you should need it?' We added a fourth question, answered on a similar five-point scale, 'How easy is it to get practical help from relatives and friends if you should need it?' Fulfilment of Fukuda ${ }^{2}$ and London $\mathrm{ME}^{19}$ diagnostic criteria were obtained using a standard checklist during baseline interview.

\section{Statistical methods}

All formal analyses were carried out using Stata version 10 on Windows XP. The sample in each case consisted of patients who had received either pragmatic rehabilitation or GP treatment as usual. Separate regression models were used to evaluate the effect of each potential moderator. First, in each regression analysis the model contained the main effects of treatment arm (coded 0 for GP treatment as usual and 1 for pragmatic rehabilitation), the putative moderator and the interaction between the moderator and the treatment arm (that is the analysis was carried out in a single step by fitting the full model with main effects and the interaction). Next, the regressions were run again with the interaction term dropped to determine the prognostic effect of the potential moderator that was common to the two groups. The outcome variable in each case was change in the Chalder Fatigue Scale scores from baseline to 1-year follow-up (70 weeks). Missing change scores were allowed for by the use of an inverse probability weight estimated through a prior logistic regression analysis using baseline information to predict which patients provided follow-up data, ${ }^{28,29}$ leading to the estimation of robust standard errors, confidence intervals and associated $P$-values. The HADS scores, SF-36 physical function, the four items of the Jenkins Sleep Scale, the social support question scores, the number of medical comorbidities and illness duration were all centred on 
their corresponding means prior to the moderator analyses, so that the main treatment effect is interpreted as the effect at the mean value of the putative moderator. However, in the moderator analyses, the main treatment effect is difficult to interpret, and it is the interaction term with pragmatic rehabilitation that is of interest in each analysis, with a statistically significant interaction term implying that the effect of pragmatic rehabilitation varies with the value of the moderator.

\section{Results}

Baseline scores or frequencies on the putative moderator variables for the entire sample are given in Table 1. Fatigue scores (Likert scored $0,1,2,3)$ for patients in the pragmatic rehabilitation and treatment as usual arms, at 0, 20 and 70 weeks, are given in Table $2 .^{15}$

Table 1 Baseline data on putative moderator variables for the entire sample $(n=296)$

\begin{tabular}{|c|c|c|c|}
\hline & $n(\%)$ & $\begin{array}{l}\text { Median } \\
\text { (IQR) }\end{array}$ & $\begin{array}{l}\text { Mean } \\
\text { (s.d.) }\end{array}$ \\
\hline Age, years & & & $44.6(11.4)$ \\
\hline Female & $230(78)$ & & \\
\hline Non-ambulatory status & $35(12)$ & & \\
\hline $\begin{array}{l}\text { EQ-5D: mobility } \\
\text { No problems } \\
\text { Some problems } \\
\text { Confined to bed }\end{array}$ & $\begin{array}{c}81(27) \\
211(71) \\
3(1)\end{array}$ & & \\
\hline $\begin{array}{l}\text { EQ-5D: self-care } \\
\text { No problems } \\
\text { Some problems } \\
\text { Severe problems } \\
\end{array}$ & $\begin{array}{c}182(62) \\
108(37) \\
5(2)\end{array}$ & & \\
\hline $\begin{array}{l}\text { EQ-5D: usual activities } \\
\text { No problems } \\
\text { Some problems } \\
\text { Severe problems }\end{array}$ & $\begin{array}{c}24(8) \\
181(61) \\
90(30)\end{array}$ & & \\
\hline Fulfilled Fukuda criteria, yes & 279 (94) & & \\
\hline Fulfilled London ME criteria, yes & $92(31)$ & & \\
\hline $\begin{array}{l}\text { Member of local ME support } \\
\text { group, yes }\end{array}$ & $58(20)$ & & \\
\hline In receipt of benefits, ${ }^{a}$ yes & $187(65)$ & & \\
\hline Any depression diagnosis, yes & $53(18)$ & & \\
\hline $\begin{array}{l}\text { OSS, }{ }^{b} \text { people close enough } \\
\text { to rely on: } n \\
\text { None } \\
\text { One or two } \\
\text { Three to five } \\
\text { More than five }\end{array}$ & $\begin{array}{c}8(4) \\
93(32) \\
110(38) \\
78(27)\end{array}$ & $3(2-4)$ & \\
\hline \multicolumn{2}{|l|}{ OSS $^{\mathrm{C}}$ concern } & $2(1-2)$ & \\
\hline \multicolumn{2}{|l|}{ OSS $^{\mathrm{C}}$ neighbours } & $3(2-4)$ & \\
\hline \multicolumn{2}{|l|}{ OSS $^{\mathrm{C}}$ relatives } & $2(1-3)$ & \\
\hline \multicolumn{2}{|l|}{ Medical comorbidities, $n$} & $1(0-2)$ & \\
\hline \multicolumn{2}{|l|}{ Illness duration, months } & $85(38-147)$ & \\
\hline $\begin{array}{l}\text { HADS }^{\mathrm{d}} \\
\text { Depression score } \\
\text { Anxiety score } \\
\text { Total score }\end{array}$ & & & $\begin{array}{c}9.6(4.1) \\
10.5(5.0) \\
20.1(8.2)\end{array}$ \\
\hline \multicolumn{3}{|l|}{ SF-36 ${ }^{\mathrm{e}}$ physical functioning, \% } & $30(18.6)$ \\
\hline \multicolumn{4}{|c|}{$\begin{array}{l}\text { ME, myalgic encephalomyelitis; OSS, Oslo Social Support Scale; HADS, Hospital Anxiety } \\
\text { and Depression Scales; SF-36, Short-Form 36-item questionnaire. } \\
\text { a. Eight patients did not answer. } \\
\text { b. Seven patients did not complete baseline OSS. } \\
\text { C. Higher OSS scores indicate less support. } \\
\text { d. Higher HADS scores indicate more depression and more anxiety. } \\
\text { e. Lower scores indicate worse functioning. }\end{array}$} \\
\hline
\end{tabular}

Table 3 shows the results of regression analyses predicting change in fatigue at 70 weeks, our primary outcome point. For ease of reading, the table reports the regression coefficients and significance values of the interaction terms only (pragmatic rehabilitation compared with treatment as usual $\times$ predictor variable). See online Table DS1 for a more detailed version of Table 3 that also includes the estimates for the main effects.

There were three significant interaction terms, showing that two baseline measures significantly moderated change in Chalder Fatigue Scale scores at 70 weeks. First, the effect of pragmatic rehabilitation (in comparison with treatment as usual) was lower in those participants who had a higher HADS depression and total scores at baseline. Second, there was a highly significant interaction between EQ-5D self-care score at baseline and treatment allocation - the effect of pragmatic rehabilitation appearing to be detrimental in those participants with severe self-care problems. However, as can be seen in Table 1, only five patients in the entire sample categorised themselves as having severe selfcare problems on this measure at baseline. The significant interaction is due to the recovery of the only patient with severe self-care problems at baseline who was allocated to treatment as usual, as compared with one patient with severe self-care problems allocated to pragmatic rehabilitation, and cannot therefore be generalised further.

In addition to the above findings, there were two trends towards significant interactions. The first of these was on the EQ-5D mobility variable, and relates to the comparison between patients self-categorised as having 'no' $v$. 'some' mobility problems. As shown in Table 4, there was a trend towards patients with no mobility problems showing greater improvements in fatigue than those with some mobility problems in the pragmatic rehabilitation arm. The opposite pattern was seen in the GP treatment as usual arm. The second trend towards a significant interaction was seen on the Oslo Social Support Scale, item 2, which is patients' reports of the amount of concern that others showed in what they were doing. Higher scores on this item represent less concern. Compared with patients allocated to treatment as usual, those allocated to pragmatic rehabilitation who reported that people showed more concern and interest in them had larger improvements in Chalder Fatigue Scale scores than those reporting that people showed less concern and interest.

The final set of analyses, where the regressions were repeated without the interaction terms, showed that three variables predicted change in Chalder Fatigue Scale scores across both the pragmatic rehabilitation and GP treatment as usual arms. Patients who were older, those who had longer baseline illness durations and those who reported severe mobility problems at baseline showed smaller improvements in fatigue. The effect with respect to severe mobility problems at baseline is produced by only three cases, and should be interpreted with caution. Table 5 shows the regression coefficients and significance values for these three variables. None of the other variables included in the analyses produced significant effects on fatigue across the two treatment arms (data not shown).

\section{Discussion}

\section{Main findings}

This study examined moderators of the effect of pragmatic rehabilitation on fatigue, as compared with GP treatment as usual, in a large RCT in primary care. The main finding was that most of the potential moderators investigated did not moderate the effects of pragmatic rehabilitation. Only baseline levels of HADS depressive symptoms and baseline total HADS scores significantly moderated the effect of pragmatic rehabilitation on fatigue at 
Table 2 Chalder Fatigue Scale scores for patients in the pragmatic rehabilitation and general practitioner treatment as usual arms, at baseline, 20 and 70 weeks

Chalder Fatigue Scale score ${ }^{\mathrm{a}}$

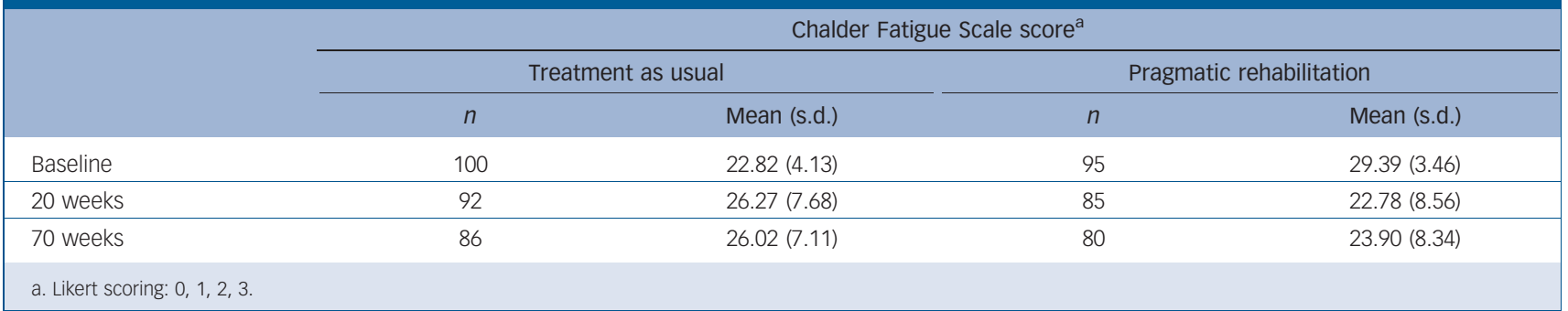

Table 3 Regression coefficients for the interaction between putative moderators and treatment in regression analyses to predict change in Chalder Fatigue Scale scores at 70 weeks $^{a}$

\begin{tabular}{|c|c|c|c|}
\hline & & moderator interact & \\
\hline & Interaction (s.e.) & $95 \% \mathrm{Cl}$ & $P$ \\
\hline Age & $-0.143(0.09)$ & -0.32 to 0.04 & 0.118 \\
\hline Gender & $1.81(2.50)$ & -3.11 to 6.73 & 0.470 \\
\hline Ambulatory status & $2.87(3.77)$ & -3.11 to 6.73 & 0.446 \\
\hline $\begin{array}{l}\text { EQ-5D mobility } \\
\text { Some problems } \\
\text { Severe problems }\end{array}$ & $\begin{array}{c}-5.55(2.84) \\
- \\
\end{array}$ & $\begin{array}{c}-11.15 \text { to } 0.05 \\
-\end{array}$ & $\begin{array}{c}0.052 \\
-\end{array}$ \\
\hline $\begin{array}{l}\text { EQ-5D self-care } \\
\text { Some problems } \\
\text { Severe problems }\end{array}$ & $\begin{array}{r}-1.89(2.35) \\
-28.72(1.73)\end{array}$ & $\begin{array}{c}-6.54 \text { to } 2.75 \\
-32.14 \text { to }-25.31\end{array}$ & $\begin{array}{r}0.422 \\
<0.001\end{array}$ \\
\hline $\begin{array}{l}\text { EQ-5D usual activities } \\
\text { Some problems } \\
\text { Severe problems }\end{array}$ & $\begin{array}{r}0.77(4.83) \\
-0.85(4.91)\end{array}$ & $\begin{array}{l}-8.76 \text { to } 10.31 \\
-10.55 \text { to } 8.56\end{array}$ & $\begin{array}{l}0.873 \\
0.863\end{array}$ \\
\hline Fulfilled Fukuda criteria & $3.95(5.28)$ & -6.48 to 14.38 & 0.455 \\
\hline Fulfilled London ME criteria & $-1.55(2.48)$ & -6.45 to 3.34 & 0.532 \\
\hline Member of local ME support group & $2.84(3.31)$ & -3.70 to 9.38 & 0.393 \\
\hline In receipt of benefits & $4.78(2.96)$ & -1.05 to 10.62 & 0.108 \\
\hline Any depression diagnosis & $-3.64(2.60)$ & -8.77 to 1.50 & 0.164 \\
\hline $\begin{array}{l}\text { Oslo Social Support Scale } \\
\text { Number of people } \\
\text { Concern }^{c} \\
\text { Neighbours }^{c} \\
\text { Relatives }^{c}\end{array}$ & $\begin{array}{l}-0.08(1.35) \\
-2.26(1.15) \\
-0.89(0.83) \\
-0.25(1.11)\end{array}$ & $\begin{array}{l}-2.75 \text { to } 2.60 \\
-4.53 \text { to } 0.01 \\
-2.54 \text { to } 0.75 \\
-2.44 \text { to } 1.95\end{array}$ & $\begin{array}{l}0.953 \\
0.051 \\
0.285 \\
0.824\end{array}$ \\
\hline Medical comorbidities, ${ }^{c} n$ & $-1.30(0.82)$ & -2.93 to 0.32 & 0.116 \\
\hline Illness duration, months ${ }^{\mathrm{c}}$ & $-0.004(0.011)$ & -0.03 to 0.02 & 0.727 \\
\hline $\begin{array}{l}\text { Hospital Anxiety and Depression Scale } \\
\text { Depression score } \\
\text { Anxiety score } \\
\text { Total score }\end{array}$ & $\begin{array}{l}-0.67(0.29) \\
-0.39(0.24) \\
-0.30(0.14)\end{array}$ & $\begin{array}{l}-1.25 \text { to }-0.10 \\
-0.86 \text { to } 0.08 \\
-0.58 \text { to }-0.02\end{array}$ & $\begin{array}{l}0.022 \\
0.107 \\
0.039\end{array}$ \\
\hline Short-Form 36-item, physical functioning ${ }^{\mathrm{C}}$ & $0.05(0.07)$ & -0.08 to 0.19 & 0.434 \\
\hline $\begin{array}{l}\text { Sleep }^{c} \\
\text { 1, Trouble falling asleep } \\
\text { 2, Wake during night } \\
\text { 3, Trouble staying asleep } \\
\text { 4, Awake feeling tired }\end{array}$ & $\begin{array}{l}-0.71(0.66) \\
-0.35(0.67) \\
-0.78(0.63) \\
-0.43(1.52)\end{array}$ & $\begin{array}{l}-2.01 \text { to } 0.60 \\
-1.68 \text { to } 0.98 \\
-2.03 \text { to } 0.47 \\
-3.43 \text { to } 2.56\end{array}$ & $\begin{array}{l}0.288 \\
0.603 \\
0.218 \\
0.775\end{array}$ \\
\hline
\end{tabular}

1-year follow-up, the primary outcome point of the trial. Patients who had any SCID-I diagnoses of depression also did less well, but this interaction did not reach statistical significance. Our second analysis showed that older age, longer illness duration and having severe mobility problems at baseline each predicted smaller changes in fatigue across the two treatment arms. The HADS depression, anxiety and total scores did not predict change in fatigue in the combined sample.

In the previous secondary care trial of pragmatic rehabilitation, baseline HADS total scores (that is depression and anxiety scores summed) moderated change in physical functioning after treatment, although the moderating effect on change in fatigue was not examined, so a direct comparison cannot be made. ${ }^{17}$ In our study, we considered HADS depression and anxiety scores separately, and found that depression but not anxiety scores interacted significantly with the effect of pragmatic rehabilitation treatment on fatigue. Our finding that a diagnosis of depression was not a significant moderator is consistent with a report that treatment effects of CBT were equivalent for patients with and without psychiatric diagnoses. ${ }^{30}$

It could be the case that patients with higher levels of depressive symptoms, although not necessarily diagnosed as 
Table 4 Change in Chalder Fatigue Scale scores for patients allocated to pragmatic rehabilitation and general practitioner treatment as usual, by initial EQ-5D mobility categorisation

\begin{tabular}{|lcc|}
\multirow{2}{*}{$\begin{array}{l}\text { Baseline EQ-5D mobility } \\
\text { categorisation }\end{array}$} & \multicolumn{2}{c}{ Mean (s.d.) } \\
\cline { 2 - 3 } No problems & $\begin{array}{c}\text { Pragmatic } \\
\text { rehabilitation }\end{array}$ & $\begin{array}{c}\text { Treatment } \\
\text { as usual }\end{array}$ \\
\hline Some problems & $7.4(9.9)$ & $0.8(6.1)$ \\
\hline
\end{tabular}

Table 5 Regression coefficients for age, baseline illness duration and EQ-5D mobility scores, in regression analyses to predict change in Chalder Fatigue Scale scores at 70 weeks across both the pragmatic rehabilitation and general practitioner treatment as usual groups

\begin{tabular}{|lllc|}
\hline & Effect (s.e.) $)^{\text {a }}$ & \multicolumn{1}{c}{$95 \% \mathrm{Cl}$} & $P$ \\
\hline Age & $-0.10(0.05)$ & -0.19 to -0.003 & 0.044 \\
\hline IIIness duration & $-0.01(0.004)$ & -0.02 to -0.003 & 0.008 \\
\hline \begin{tabular}{llll|} 
EQ-5D mobility \\
Some problems
\end{tabular} & $-0.30(1.47)$ & -3.20 to 2.59 & 0.836 \\
$\quad$ Severe problems & $-2.95(1.29)$ & -5.51 to -0.40 & 0.024 \\
\hline & & \\
\hline a. Unstandardised regression coefficient. & & \\
\hline
\end{tabular}

depressed, are unable to benefit from pragmatic rehabilitation unless their depressive symptoms, particularly loss of interest, pleasure and motivation, are treated first. In such cases, it would be necessary to explain the rationale for the treatment approach carefully, so that patients were assured that their CFS/ME symptoms were understood and not being misdiagnosed as depression. Alternatively, these patients might require longer or more intensive treatment with pragmatic rehabilitation than was possible in our trial, or pragmatic rehabilitation may require some modifications, for example, by including a wider variety of behavioural activation or emotion regulation elements, to address the additional difficulties of patients who have higher levels of depressive symptoms. In line with this suggestion, a recent meta-analytic review of CBT and GET for CFS/ME found that, although both treatments are effective for CFS/ME, CBT, which contains components that might explicitly address emotional difficulties, is the more effective treatment for patients with comorbid anxiety or depressive disorders. ${ }^{3}$

We previously suggested that the smaller effect of pragmatic rehabilitation seen in the primary care FINE trial ${ }^{13}$ when compared with the earlier secondary care trial, ${ }^{14}$ might have been due to the sample in the primary care trial having a longer illness duration, greater levels of disability and complicating medical comorbidities. Contrary to our expectations, in the moderator analysis, illness duration did not moderate the effect of pragmatic rehabilitation treatment, although our second set of analyses showed that longer illness duration was a predictor of poorer outcome at 70 weeks, across the two treatment arms.

The picture with respect to level of disability is more complicated. There was no moderating effect on response to pragmatic rehabilitation of baseline SF-36 physical functioning scores, nor of ambulatory status, defined as needing a mobility aid on most days. However, on the EQ-5D mobility question, patients who initially categorised themselves as having some mobility problems showed a smaller improvement in fatigue with pragmatic rehabilitation than did those who initially categorised themselves as having no problems. Our second analyses showed that, across the two treatment groups, three patients with severe mobility problems at baseline showed a smaller improvement in fatigue at 70 weeks. Finally, the number of medical comorbidities experienced by patients in our study did not moderate the effect of pragmatic rehabilitation on fatigue, nor did it significantly predict outcome across the two treatment groups combined.

Our perception that the patients in the FINE trial had complex social needs that might have hampered their ability to respond to treatment is borne out to some extent by the trend for those patients who perceived themselves as less well supported by others benefitting less from pragmatic rehabilitation. This tentative finding underlines the importance of considering social factors when GPs and primary care teams are working with patients with CFS/ME; for example, primary care clinicians may suggest referral for social care assessment or to a local community organisation for patients with complex needs. Our study did not replicate previous findings from secondary care studies that patients in receipt of benefits, or those who belonged to self-help groups benefitted less from treatment. Finally, age and gender did not moderate the effect of pragmatic rehabilitation on fatigue.

\section{Strengths and limitations}

The FINE trial was the first UK study of treatments for CFS/ME delivered by non-specialists in primary care. Pragmatic rehabilitation had a relatively modest, although, we would argue, still clinically significant effect on fatigue at the 1-year follow-up point, but not on other outcomes; this has limited the analyses we were able to carry out here. Our findings need to be interpreted with caution: we investigated the potential moderating effects of over 20 baseline variables, although most of these were prespecified in our protocol, ${ }^{16}$ and our two positive findings may be type 1 errors.

\section{Implications}

Further research to replicate the finding that level of depressive symptoms moderates the effect of pragmatic rehabilitation treatment on fatigue is needed before we can have confidence in the result. Additionally, our findings suggest that future research to determine the optimal length and intensity of pragmatic rehabilitation for those with different levels of depressive symptoms would be useful. The effect of including additional elements in the pragmatic rehabilitation programme to address mood or emotional difficulties should also be examined.

The overall conclusion from our study is that pragmatic rehabilitation delivered in primary care by non-specialists will be a helpful treatment, particularly for those patients who are well supported and not overwhelmed with emotional difficulties. For patients with more complex needs, it may be necessary to include additional elements in the pragmatic rehabilitation programme. Alternatively, patients with mood disorders or with high levels of depressive symptoms may require treatment with CBT. ${ }^{3}$

\section{Funding}

The FINE trial (IRCTN74156610) was funded by the UK Medical Research Council (Grant number G200212) and the UK Department of Health.

A. J. Wearden, PhD, School of Psychological Sciences, University of Manchester, Manchester; G. Dunn, PhD, School of Community Based Medicine, University of Manchester; G. Dunn, PhD, School of Community Based Medicine, University of
Manchester, Manchester; C. Dowrick, MD, Institute of Psychology, Health and Society, University of Liverpool, Liverpool; R. K. Morriss, MD, School of Community Health Sciences, University of Nottingham, Nottingham, UK

Correspondence: A. J. Wearden, University of Manchester, School of Psychological Sciences, Coupland 1 Building, Oxford Road, Manchester M13 9PL, UK. Email: Alison.wearden@manchester.ac.uk

First received 10 Dec 2011, final revision 11 May 2012, accepted 29 May 2012 


\section{References}

1 Sharpe MC, Archard LC, Banatvala JE, Borysiewicz LK, Clare AW, David A, et al. A report - chronic fatigue syndrome: guidelines for research. $J R$ Soc Med 1991; 84: 118-21.

2 Fukuda K, Straus SE, Hickie I, Sharpe MC, Dobbins JG, Komaroff A, et al. The chronic fatigue syndrome - a comprehensive approach to its definition and study. Ann Intern Med 1994; 121: 953-9.

3 Castell BD, Kazantzis N, Moss-Morris RE. Cognitive behavioral therapy and graded exercise for chronic fatigue syndrome: a meta-analysis. Clin Psycho 2011; 18: 311-24.

4 Malouff JA, Thorsteinsson EB, Rooke SE, Bhullar N, Schutte NS. Efficacy of cognitive behavioral therapy for chronic fatigue syndrome: a meta-analysis Clin Psychol Rev 2008; 28: 736-45.

5 Price JR, Mitchell E, Tidy E, Hunot V. Cognitive behaviour therapy for chronic fatigue syndrome in adults. Cochrane Database Syst Rev 2008; 3: CD001027.

6 Chambers D, Bagnall AM, Hempel S, Forbes C. Interventions for the treatment, management and rehabilitation of patients with chronic fatigue syndrome/myalgic encephalomyelitis: an updated systematic review. J $R$ Soc Med 2006; 99: 506-20.

7 Edmonds M, McGuire $\mathrm{H}$, Price J. Exercise therapy for chronic fatigue syndrome. Cochrane Database Syst Rev 2004; 3: CD003200.

8 White PD, Goldsmith KA, Johnson AL, Potts L, Walwyn R, DeCesare JC, et al Comparison of adaptive pacing therapy, cognitive behaviour therapy, graded exercise therapy, and specialist medical care for chronic fatigue syndrome (PACE): a randomised trial. Lancet 2011; 377: 823-36.

9 Department of Health. A Report of the CFS/ME Working Group: Report to the Chief Medical Officer of an Independent Working Group. Department of Health, 2002.

10 National Institute for Health and Clinical Excellence. Chronic Fatigue Syndrome/Myalgic Encephalomyelitis (Or Encephalopathy): Diagnosis and Management. Clinical Guideline CG53. NICE, 2007.

11 Wearden AJ, Chew-Graham C. Managing chronic fatigue syndrome in UK primary care: challenges and opportunities. Chronic Illn 2006; 2: 143-53.

12 Raine $R$, Haines A, Sensky $T$, Hutchings A, Larkin $K$, Black N. Systematic review of mental health interventions for patients with common somatic symptoms: can research evidence from secondary care be extrapolated to primary care? BMJ 2002; 325: 1082-5.

13 wearden AJ, Dowrick C, Chew-Graham C, Bentall RP, Morriss RK, Peters S, et al. Nurse led, home based self help treatment for patients in primary care with chronic fatigue syndrome: randomised controlled trial. BMJ 2010; 340 : c17777.

14 Powell P, Bentall RP, Nye FJ, Edwards KHT. Randomised controlled trial of patient education to encourage graded exercise in chronic fatigue syndrome. BMJ 2001; 322: 387-90.
15 Wearden AJ, Dowrick C, Chew-Graham C, Bentall RP, Morriss RK, Peters S, et al. Rapid response: Fatigue Scale. BMJ 2010; 340: c1777.

16 Wearden AJ, Riste L, Dowrick C, Chew-Graham C, Bentall RP, Morriss RK, et al. Fatigue intervention by nurses evaluation - the FINE trial. A randomised controlled trial of nurse led self-help treatment for patients in primary care with chronic fatigue syndrome: study protocol [ISRCTN74156610]. BMC Med 2006; 4: 9.

17 Bentall RP, Powell P, Nye FJ, Edwards RHT. Predictors of response to treatment for chronic fatigue syndrome. Br J Psychiatry 2002; 181: 248-52.

18 Emsley R, Dunn G, White IR. Mediation and moderation of treatment effects in randomised controlled trials of complex interventions. Stat Methods Med Res 2010; 19: 237-70.

19 Dowsett EG, Goudsmit E, Macintyre A, Shepherd C. London Criteria for ME: Report from the National Task Force on Chronic Fatigue Syndrome, Post Viral Fatigue Syndrome and Myalgic Encephaolmyelitis. Westcare, 1994.

20 Shephard RJ, Bailey DA, Mirwald RL. Development of Canadian home fitness test. Can Med Assoc J 1976; 114: 675-9.

21 Chalder T, Berelowitz G, Pawlikowska T, Watts L, Wessely S, Wright D, et al. Development of a fatigue scale. J Psychosom Res 1993; 37: 147-53.

22 Williams A. EUROQOL - a new facility for the measurement of health-related quality-of-life. Health Policy 1990; 16: 199-208.

23 Ware JE, Sherbourne CD. THE MOS 36-item short-form health survey (SF-36).1. Conceptual framework and item selection. Med Care 1992; 30 473-83.

24 Jenkins CD, Stanton BA, Niemcryk SJ, Rose RM. A scale for the estimation of sleep problems in clinical research. J Clin Epidemiol 1988; 41: 313-21.

25 Zigmond AS, Snaith RP. The hospital anxiety and depression scale. Acta Psychiatr Scand 1983; 67: 361-70.

26 First MB, Spitzer RL, Gibbon M, Williams JBW. Structured Clinical Interview for DSM-IV-TR-Axis I Disorders, Research Version, Non-patient Edition (SCID-I/ NP). Biometrics Research, New York State Psychiatric Institute, 2002.

27 Dalgard OS, Dowrick C, Lehtinen V, Vazquez-Barquero JL, Casey $P$ Wilkinson G, et al. Negative life events, social support and gender difference in depression - a multinational community survey with data from the ODIN study. Soc Psychiatry Psychiatr Epidemiol 2006; 41: 444-51.

28 Heyting A, Tolboom J, Essers JGA. Statistical handling of drop-outs in longitudinal clinical-trials. Stat Med 1992; 11: 2043-61.

29 Everitt B, Pickles A. Statistical Aspects of the Design and Analysis of Clinical Trials. Imperial College Press, 2004

30 Prins J, Bleijenberg G, Rouweler EK, van der Meer J. Effect of psychiatric disorders on outcome of cognitive-behavioural therapy for chronic fatigue syndrome. Br J Psychiatry 2005; 187: 184-5. 